\title{
Proposing a Formal Model for Performance Improvement in Grid Environment
}

\author{
Samira Amirshekari \\ Department of Computer \\ Engineering \\ Islamic azad university,Malayer \\ branch,Malayer,Iran
}

\author{
Ali Harounabadi \\ Department of Computer \\ Engineering \\ Islamic azad university, Central \\ Tehran branch,Tehran,Iran
}

\begin{abstract}
The cooperation of the geographical distributed resources for solving the great problems is called the computing grid. An efficiency scheduling system is necessary to reach the aim. In the proposed method, RMS divides every task in to several subtasks. Then, the appropriate resources which can create the more reliability and lower execution time for the task were chosen in the system. It means the reliability and execution time parameters were optimized in the proposed model. In the method, the operator can distinguish the Importance of the parameters in the system for choosing the resources until the choosing is conducted considering the significance degree. Multiple attribute decision making (MADM) were used to choose the resources optimumly. In this paper, the task scheduling by RMS and the task execution within grid resources are modeled using coloured Petri nets.In addition, an example of proposed model for a sample grid environment is constructed and analysed using CPN Tools. The results demonstrated reliability improvement compared to the previous model.
\end{abstract}

\section{Keywords}

Computing grid ,task scheduling, coloured petri nets, reliability, resources management system.

\section{INTRODUCTION}

A computational grid is defined as a hardware and software infrastructure, which provides dependable, consistent, pervasive, and inexpensive access to computational resources existing on the network [1] . It is as a common environment which is configurated by standard and permanent services establishent. Computing grid tries to solve the scientific timeconsuming and complicated problems optimumly using the computers processing power presented in network and the resources sharing. In order to reach to grid aim and maximum usage of present resources in the grid environment, the subtask distribution style among the resources and their scheduling is considerably important. Breaking the tasks in to subtasks and their distribution among the resources in the grid environment is in charge of the Resources Management System. The system catches the tasks from the operators and divide them into subtask and then distributed the subtasks among the resources regarding to their characteristic and the resources properties. The tasks allocation to the resources in the recognized intervals is called the task scheduling. The scheduling algorithms undertake the subtasks distribution duty among the grid resources. The scheduling is conducted considering the quality of service and it is tried the subtasks are distributed among the resources in the scheduling time as maximum QoS will be achieved[2-4].

The reliability is one of the important QoS parameters that causes the efficiency improvement in the grid environment[5]. In the environments which the task execution time or the resource service time is significant, the system reliability is defined as the probability in which a task results are produced in a time lower than a specific time[6-10]. Usually, the necessity of reliability increase is redundancy in the resources allocation to the tasks. The work which has previously conducted in the field [11] is the random task allocation to the resources, but in order to achieve better result, the most appropriate resource can be chosen for every task. Therefore, in the research it has tried to use a high level formal model to present the model in addition to consider execution time and reliability improvment.

In section2, some information are presented about the grid and petri nets background, and related work is introduced in Section 3, in section4, the proposed model and the task allocation style to resources are described. In section 5 , the proposed method is evaluated and the last section includes the conclusion.

\section{BACKGROUND}

The grid aims to provide a field which the operator can use the information and facilities of many heterogeneous resources presented in network. The aim is conducted by the resource management system(RMS). There are different topologies for the resources in the grid environment . we have used star topology in which RMS locates in the resources center (Fig1).

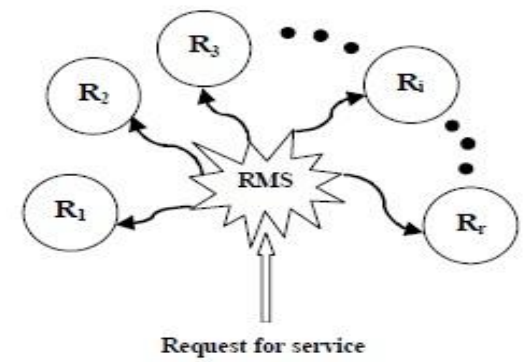

Fig1: star topology of the grid environment

The petri nets are dynamic system which deal with meanings such as concurrency, synchronization and mutual exclusion 
and are generally as distributed environments. Petri nets are a tool for modeling and are used to model the synchronized, parallel, dynamic and detached system and provide further description power comparing to queue net. Petri nets possess formal behavior and structure, also they have the graphical show capability, then they can easily make model. The coloured petri nets is a 9-tuple as following:

\section{$\mathrm{CPN}=\left(\sum, \mathrm{P}, \mathrm{T}, \mathrm{A}, \mathrm{N}, \mathrm{C}, \mathrm{G}, \mathrm{E}, \mathrm{I}\right):$}

- $\quad \sum$ is a finite set of non-empty types, also called colour sets.

- $\quad \mathrm{P}$ is a finite set of places.

- $\quad \mathrm{T}$ is a finite set of transitions.

- $\quad \mathrm{A}$ is a finite set of arcs such that: $\mathrm{P} \cap \mathrm{T}=\mathrm{P} \cap \mathrm{A}=\mathrm{T} \cap \mathrm{A}=\varnothing$.

- $\quad \mathrm{N}$ is a node function. It is defined from $\mathrm{A}$ into $(\mathrm{T} \times \mathrm{P}) \cup($ $\mathrm{P} \times \mathrm{T}$ ).

- $\quad \mathrm{C}$ is a colour function. It is defined from $\mathrm{P}$ into $\sum$.

- $\mathrm{G}$ is a guard function. It is defined from $\mathrm{T}$ into expressions such that $\forall \mathrm{t} \in \mathrm{T}$ :[Type $(\mathrm{G}(\mathrm{t}))=\mathrm{B} \wedge$ Type $\left(\operatorname{Var}\left(\mathrm{G}(\mathrm{t}) \subseteq \sum\right]\right.$ B to denote the Boolean type.

- $\quad \mathrm{E}$ is an arc expression function. It is defined from $\mathrm{A}$ into expressions such that: $\forall \mathrm{a} \in \mathrm{A}$ : [Type $(\mathrm{E}(\mathrm{a}))=\mathrm{C}(\mathrm{p})_{\mathrm{MS}} \wedge$ Type $\left.(\operatorname{Var}(E(a))) \subseteq \sum\right]$ where $p$ is the place of $N(a)$.

- $\quad \mathrm{I}$ is an initialisation function. It is defined from $\mathrm{P}$ into closed expressions such that: $\forall p \in P$ :[Type $(\mathrm{I}(\mathrm{p}))=$ $\left.\mathrm{C}(\mathrm{p})_{\mathrm{MS}}\right]$.

CPN Tools [12] is one of the tools which make modeling, validity check and coloured petri nets analysis possible.

\section{RELATED WORK}

One of the most important affairs in the computing grid is resources scheduling and allocation in the system from past to now, the researchers and sciences have had further efforts in the field of the net science and computing grid. Many works have conducted in the field of grid, but the majority of them have related to the scheduling modeling and workflow or optimized the service quality parameters. Some of the efforts are as following :

Meibodi. M et al. proposed a resources scheduling schema for optimizing the resources scheduling in the computing grid which is located on 3 layer( home, local, grid) according to the requests categorization. Every layer has special function for receiving and delivering the subtask to its upper or lower layer. The schema has modeled and analyzed through Hierarchical Stochastic Petri Nets (HSPN)[13].

Li.L et al. accomplished the tasks scheduling modeling in the grid environment using the queue system ,they proposed a multiserver multiqueue (MSMQ) based scheduling system and they presented the modeling as high level formal using a Stochastic Petri Nets. Then they evaluates the throughput of the service grid. [14].
Han. Y et al. presented a resource scheduling algorithm called XMin-min . In the XMin-min algorithm, they considered not only the expected execution time of tasks, but also expected communication time when calculating expected completion time. they proposed the execution cost of tasks and budget of application are selected as QoS and an algorithm XMin-min with QoS. they construct a simple model for the resource scheduling in grid computing using EHLTPN(Extended HighLevel Timed Petri Net) [15].

Dai.Y-Sh et al. calculated the reliability in the grid services. The researchers considered the computing grid system in which resources management system divides the tasks into subtasks and deliver the subtasks to different resources for parallel execution. Also ,they presented a fast numerical algorithm for their evaluation for arbitrary subtask distribution in grid with star architecture [6].

Parsa. S et al. presented a mathematical solution based on queuing theory and a generalized stochastic Petri net model to minimize the total makespan of the grid computing environments. In the proposed models was considered both grid and local tasks. The aim of the modeling was to find subtask arrival rates at each of the grid resources to reach minimum total makespan [16].

Azgomi. $\mathrm{M}$ et al. proposed CPN-based modeling pattern formally that it describes the process of task distribution and execution within the grid environment. They have also proposed a method for evaluation the grid service reliability based on the analysis of the model.The researchers allocated the subtasks to the resources randomly [11]. While, we choose the resources based on the execution time and reliability for achieving better result

\section{PROPOSED METHOD}

In section (4.1) the subtasks scheduling in RMS and subtask execution in the resources are modeled and reliability is evaluated and in section (4.2) the subtasks allocation style to the resources are studied.

\subsection{Subtasks Scheduling and Execution}

It is supposed that the grid environment possesses the star topology and RMS is relation to all resources presented in the grid. RMS is so fast and reliable.After receiving the task from the operator, RMS divides into subtasks. After the division, RMS allocates every subtask to more one resource in order to increase the reliability of the task execution which the redundancy technique was used. But every resource just processes a subtask. As soon as the resources receive the necessary information from RMS, the subtask processing is done. After dividing to subtask, table called selection table including execution time estimation information and probability of having no failure in resources is immediately produce for every subtask for every resource. The subtask modelling is accomplished according to figure 2 . 


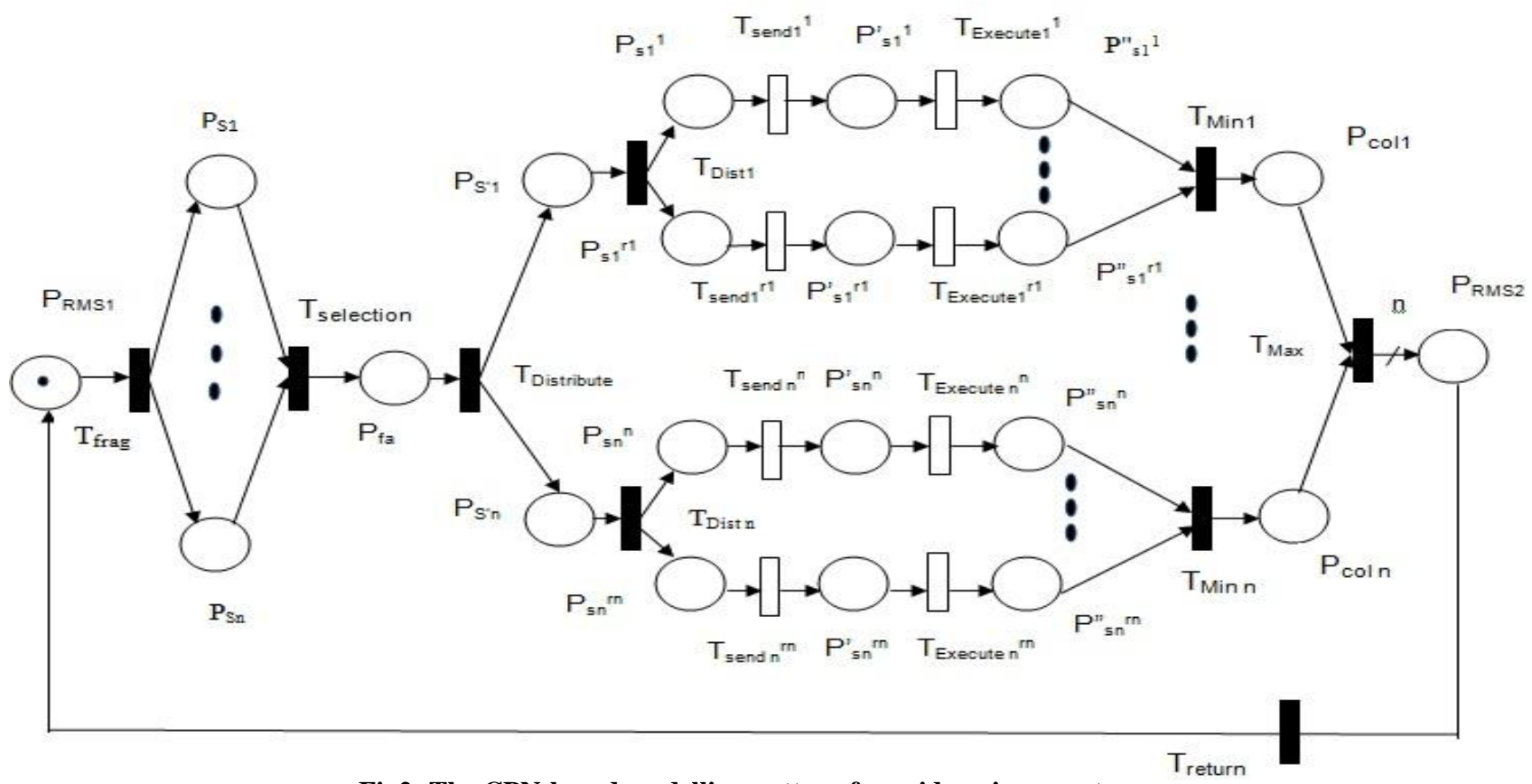

Fig2: The CPN-based modelling pattern for grid environment

Regarding to [11], the operators deliver their requests to RMS and after placing the token in RMS, transition Frag fires untile the task divide into subtask. In the stage, a subtask is allocated to several resources based on the redundancy technique. In [11], the tasks were randomly allocated to the resources, but in the proposed model the tasks are allocated to the best resource both based on execution time and the reliability. So, the subtasks enter to selection subnet until the most appropriate resources are chosen for them. The choice process will be described subsequently. After firing transmissions Distribute and Selection, the tokens are located in places $\mathrm{P}_{\mathrm{si}}{ }^{\mathrm{j}}$. The transmissions $\mathrm{T}_{\text {Dist }}, \mathrm{T}_{\mathrm{Frag}}, \mathrm{T}_{\text {Distribute }}$ and $\mathrm{T}_{\text {Selection }}$ are done rapidly and continuous

Tokens carry the probabilities in addition to time. The probability the communications link doesn't fail between resource $\mathrm{j}$ and RMS during data transmission is shown with $\mathrm{q}_{\mathrm{j}}\left(\tau_{i j}\right)$ and the amount is added to token during passing from transmission $\mathrm{T}_{\text {sendi }}{ }^{\mathrm{j}}$, also the probability resource $\mathrm{j}$ doesn't fail during subtask execution is shown with $\mathrm{p}_{\mathrm{j}}\left(\mathrm{T}_{\mathrm{ij}}\right)$, the amount is added to tokens during passing from transmission $T_{\text {Execute } i}{ }^{j}$. the amounts are calculated as followings:

If the necessary data for exchanging between resource $R_{j}$ and RMS for subtask $j$ execution is supposedly equal to $a_{i}$ and the communications link bandwidth between RMS and resource $R_{j}$ is shown with $b w_{j}$, then the data transmission time between RMS and resource $R_{j}$ is calculated as formula1:

$\tau_{\mathrm{ij}}=\frac{a_{\mathrm{i}}}{b w_{j}}$

If the whole necessary calculation volume for the task execution to RMS equals to $c$ and the number of total resources connected to RMS equals to n, RMS divides task $\mathrm{S}$ to $\mathrm{s}_{1}, \mathrm{~s}_{2}, \mathrm{~s}_{3}, \ldots \mathrm{s}_{\mathrm{n}}$ that every subtask has the calculational volume in size of $c_{j}$ according to formula (2): $\sum_{j=1}^{n} c_{j}=c$

If resource $R_{j}$ processing speed is shown with $\mathrm{ps}_{j}$, then subtask $s_{i}$ processing time by resource $R_{j}$ is achieved from formula(3):

$\mathrm{T}_{\mathrm{ij}}=\frac{c_{\mathrm{f}}}{\mathrm{p} s_{\mathrm{f}}}$

The execution time of a subtask equals to summation of times $\mathrm{T}_{\mathrm{ij}}$ and $\tau_{\mathrm{ij}}$ and and also the probability equals to the multiplications of $p_{i \times j} \times q_{i \times j}$ probabilities. The tokens which passes from transmission $T_{\text {Execute } i} \mathrm{i}$ carry $\left(\left(\tau_{i j}+T_{i j}\right),\left(p_{i j} \times q_{i j}\right)\right)$ couple which represents the successful execution time and its probability. When the subtask execution is finished, transmission Min is fired until the least $s_{\mathrm{i}}$ execution time, from the resources which have allocated to the subtask, is calculated

Since the redundancy technique is used for reliability enhancement, one subtask is processed by several resources, simultaneously. Therefore, if the subtask $\mathrm{s}_{\mathrm{i}}$ is assigned to the resources composing set $\mathrm{R}_{\mathrm{ri}}$, then the total time of the subtask execution $\left(T_{\mathrm{i}} R_{\mathrm{Ff}}\right)$ will be equal to:

$$
\begin{aligned}
& T_{\mathrm{i} R_{\mathrm{rf}}}=\min \left(\tau_{\mathrm{ij}}+T_{\mathrm{ij}}\right) \\
& \mathrm{j} \in R_{\text {Yi }}
\end{aligned}
$$

Then , transmission Max calculates total time of task execution which has entered to RMS. Since, the whole subtask are executed as cocurrency, the execution time related to the most time consuming subtask will be equaled to the execution time of total task. The total time of task execution (T ) can be computed by the following equation:

$$
\mathrm{T}=\max _{1<\mathrm{i}<\mathrm{n}}\left(T_{\mathrm{i} i R_{\mathrm{Pf}}}\right)
$$


Then other task is entered to RMS system through firing transmission $\mathrm{T}_{\text {return }}$.

The reliability of the task execution in the grid environment is calculated as formula (6).in the equation, i represents total achieved $T$ and $P_{i}$ is the probability of the task execution in $T_{i}$ time and $\mathrm{T}^{*}$ is the same $\mathrm{T}_{\text {reliable }}$ time. Also, 1 (condition) is a function which is defined as following.

$\mathrm{R}_{\mathrm{T}}^{*}=\sum_{T=1}^{1} P_{I^{*}}\left(T_{I<} T^{*}\right)$

$1($ true $)=1,1($ false $)=0$

The grid environment reliability is defined as the probability of a task results production in a time lower than distinguished $\mathrm{T}_{\text {reliable }}$ time. The hypothesis has also used for evaluation.

\subsection{The subtasks allocation style to resources}

In the proposed model, multiple attribute decision making (MADM) models are used to allocate the subtasks to the resources which the models are applied to choose the superior alternative . usually, MADMs are formulated by following $\operatorname{matrix}($ Table1)[17].

\section{Table1. multiple attribute decision making matrix}

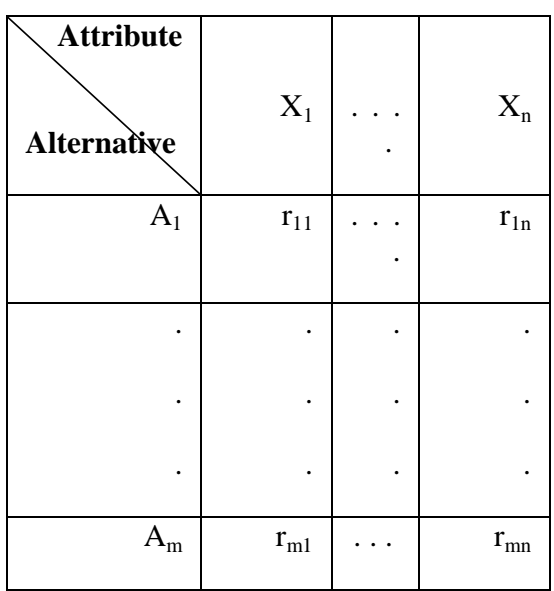

As $A_{i}$ indicates ith alternative and $x_{j}$ indicates $j$ th attribute and $r_{i j}$ represents $j$ th attribute value for ith alternative. The considered matrix is selection table including the estimation of the execution time and subtasks reliability(probability having no failure). In selection subnet, the following operation is accomplished.

Compensatory model was used to process the information from a MADM problem. In the models, the exchange among the attributes is permitted as the weak point presented in a attribute is compensated by benefit presented in the other attribute. One of the compensation methods is hierarchial additive weighting method until the most appropriate is chosen. For us, the first level includes the unique goal of decision making with unit superiority(unit scaler). The second level of serial decision making includes two attributes(probability having no failure and execution time) presented and effected from the decision making goal which the superiority vector $\left(\mathrm{w}^{2}\right)$ is one of the most appropriate methods that is calculated regarding to the decision goal. The third level includes the given alternatives from the problem which every of them is effected by every attributes presented in second level.

Their superiority matrix $\left(\mathrm{w}^{3}\right)$ should be calculated in return for every variables(attributes) presented in second level. Then ,the superiority vector for the lowest level (alternatives) toward total decision making system is :

superiority vector from the lowest level $=\mathrm{w}=\mathrm{w}^{3} \cdot \mathrm{w}^{2} .1$

for calculating the alternatives weights in return for each attribute, the digits of every column should firstly be normalized $\left(\mathrm{r}_{\mathrm{ij}}\right)$. For the execution time $\left(\mathrm{T}_{\text {execution }}\right)$ which is the negative aspect, the calculation is done through formula(8) and for the probability having no failure $\left(\mathrm{P}_{\text {no failure }}\right)$ which is positive aspect, the calculation is done through formula(9).

$\mathrm{C}_{\mathrm{i}}=\frac{\frac{1}{\mathrm{r}_{\mathrm{i}}}}{\sum_{\mathrm{i}=-1}^{\mathrm{n}} \frac{1}{\mathrm{r}_{\mathrm{i}}}}$

$C_{\mathrm{ij}}=\frac{\mathrm{r}_{\mathrm{ij}}}{\sum_{\mathrm{i}=1}^{\mathrm{n}} \mathrm{r}_{\mathrm{ij}}}$

Now, entropy method is used to achieve vector $\mathrm{w}^{2}$.

In the method, selection matrix is firstly calculated as $\left(\mathrm{P}_{\mathrm{ij}}\right)$ by formula(10)

$p_{\mathrm{ij}}=\frac{\mathrm{r}_{\mathrm{ij}}}{\sum_{\mathrm{i}=1}^{\mathrm{m}} \mathrm{r}_{\mathrm{ij}}} \forall i_{i j} j$

It will be for $E_{j}$ from $P_{i j}$ collection, in return for every characteristic according to formula (11).

$\mathrm{E}_{\mathrm{j}}=-\frac{1}{\ln m} \sum_{\mathrm{i}=1}^{m}\left[p_{\mathrm{i} j} \cdot \operatorname{Ln} p_{\mathrm{ij} j}\right]: \forall j$

For deviation $\left(d_{j}\right)$ from produced information in return jth attribute is according to formula (12):

$\mathrm{d}_{\mathrm{j}}=1-\mathrm{E}_{\mathrm{j}} ; \forall \forall j$

and finally, it will be for weights $\left(\mathrm{w}_{\mathrm{j}}\right)$ of the present attributes.

$w_{j}=\frac{d_{j}}{\sum_{j=1}^{n} d_{j}}: \forall j$

Operator can have a mental judgment $\left(\lambda_{\mathrm{j}}\right)$ as the relative importance of the attribute for jth attribute. Meaning, we can recognize the significance of $\mathrm{T}_{\text {execution }}$ and $\mathrm{P}_{\text {no failure }}$ attributes. $\mathrm{W}^{\prime}$ is calculated through formula 14

$\mathrm{w}^{\prime}=\frac{\lambda_{\mathrm{j}} \mathrm{w}_{\mathrm{j}}}{\sum_{\mathrm{m}}^{\mathrm{n}} \lambda_{\mathrm{j}} \mathrm{w}_{\mathrm{j}}} \quad \forall \mathrm{j}$

Now, regarding formula(7), we multiply the achieved matrix from the normalization of the selection table digits to weights matrix. New matrix shows the superiority scale of the appropriate resources and the resource which has the most amount shows the most appropriate alternative. 
CPN model of the resources choice which has simulated by CPN Tools is presented in next section.

\section{EVALUATION OF PROPOSED METHOD}

In this section, an example is presented to clarify the matter. Suppose there are four resources in the grid environment. Every resource has its bandwidth, processing speed, probability having no failure which are shown in the following tables(Table2,4,5). Suppose RMS divides the enterned task in to two subtasks and then using MADM , that was descriped above, chooses two of the most appropriate resources for every subtask regarding to the $T_{\text {execution }}$ and $\mathrm{P}_{n o}$ failure. Suppose the subtask that has more data is subtask1. Selection table is shown in Table6.

Table2. processing speed and bandwidth

\begin{tabular}{|l|r|r|r|r|}
\hline & R1 & R2 & R3 & R4 \\
\hline $\begin{array}{l}\text { Processing } \\
\text { speed } \\
\text { (MIPS) }\end{array}$ & 40 & 60 & 3 & 8 \\
\hline $\begin{array}{l}\text { bandwidth } \\
\text { (Mbps) }\end{array}$ & 30 & 55 & 4 & 5 \\
\hline
\end{tabular}

Table3. subtask characteristics

\begin{tabular}{|l|r|r|}
\hline & S1 & S2 \\
\hline $\begin{array}{l}\text { Computational } \\
\text { complexity } \\
(\mathrm{MI})\end{array}$ & 60 & 100 \\
\hline $\begin{array}{l}\text { Required data } \\
(\mathrm{Mb})\end{array}$ & 300 & 80 \\
\hline
\end{tabular}

Table4. probabilities of having no failure in the resources during processing $(p)$

\begin{tabular}{|r|r|r|r|r|}
\hline & R1 & R2 & R3 & R4 \\
\hline S1 & 0.95 & 0.64 & 0.92 & 0.60 \\
\hline S2 & 0.94 & 0.63 & 0.87 & 0.67 \\
\hline
\end{tabular}

Table5. probabilities of having no failure in the links during data transmission $(q)$

\begin{tabular}{|r|r|r|r|r|}
\hline & R1 & R2 & R3 & R4 \\
\hline S1 & 0.98 & 0.56 & 0.88 & 0.58 \\
\hline S2 & 0.91 & 0.60 & 0.93 & 0.61 \\
\hline
\end{tabular}

Table6. selection table

\begin{tabular}{|c|c|c|c|c|}
\hline \multicolumn{3}{|c|}{ Subtask1 } & \multicolumn{2}{c|}{ Subtask2 } \\
\hline & $\mathrm{T}_{\text {execution }}$ & $\mathrm{P}_{\text {no failure }}$ & $\mathrm{T}_{\text {execution }}$ & $\mathrm{P}_{\text {no failure }}$ \\
\hline R1 & 11.5 & 0.93 & 5.1 & 0.85 \\
\hline R2 & 6.4 & 0.35 & 3.1 & 0.37 \\
\hline R3 & 95 & 0.80 & 53.3 & 0.80 \\
\hline R4 & 67.5 & 0.34 & 28.5 & 0.4 \\
\hline
\end{tabular}

It is considered the significance degree of $\mathrm{P}_{\mathrm{no}}$ failure 0.6 (Because it is more important) and the $\mathrm{T}_{\text {execution }} 0.4$, then the appropriate resource are firstly chosen regarding to $\mathrm{T}_{\text {reliable }}$ and after that the hierarchial additive weighting method. The appropriate resource is firstly chosen to s1 and then s2 Afterward the second appropriate resource is chosen to $\mathrm{s} 2$ and s1. If it has yet remained a subtask which the second appropriate resource was not chosen for it, the resource is chosen among the other remined resources using the hierarchial additive weighting method.

When two of the best resources are chosen for every subtask $\mathrm{s} 1$ and s2, as it was mentioned a resource which possesses lower execution time is chosen among two resources.

For example, using the method ,if $\mathrm{T}^{*}=40$ then resource R1 and $\mathrm{R} 3$ are chosen to $\mathrm{s} 1$ and $\mathrm{R} 2$ and $\mathrm{R} 4$ to $\mathrm{s} 2$. In this case, task execution time is 11.5 and task reliability is 0.578 ( Regarding to this example ,for $\mathrm{T}^{*}=40$, it is the best reliability).

Figure 3 shows CPN model and figure4 shows the subnet of optimal resources choice which was simulated using CPN Tools. 


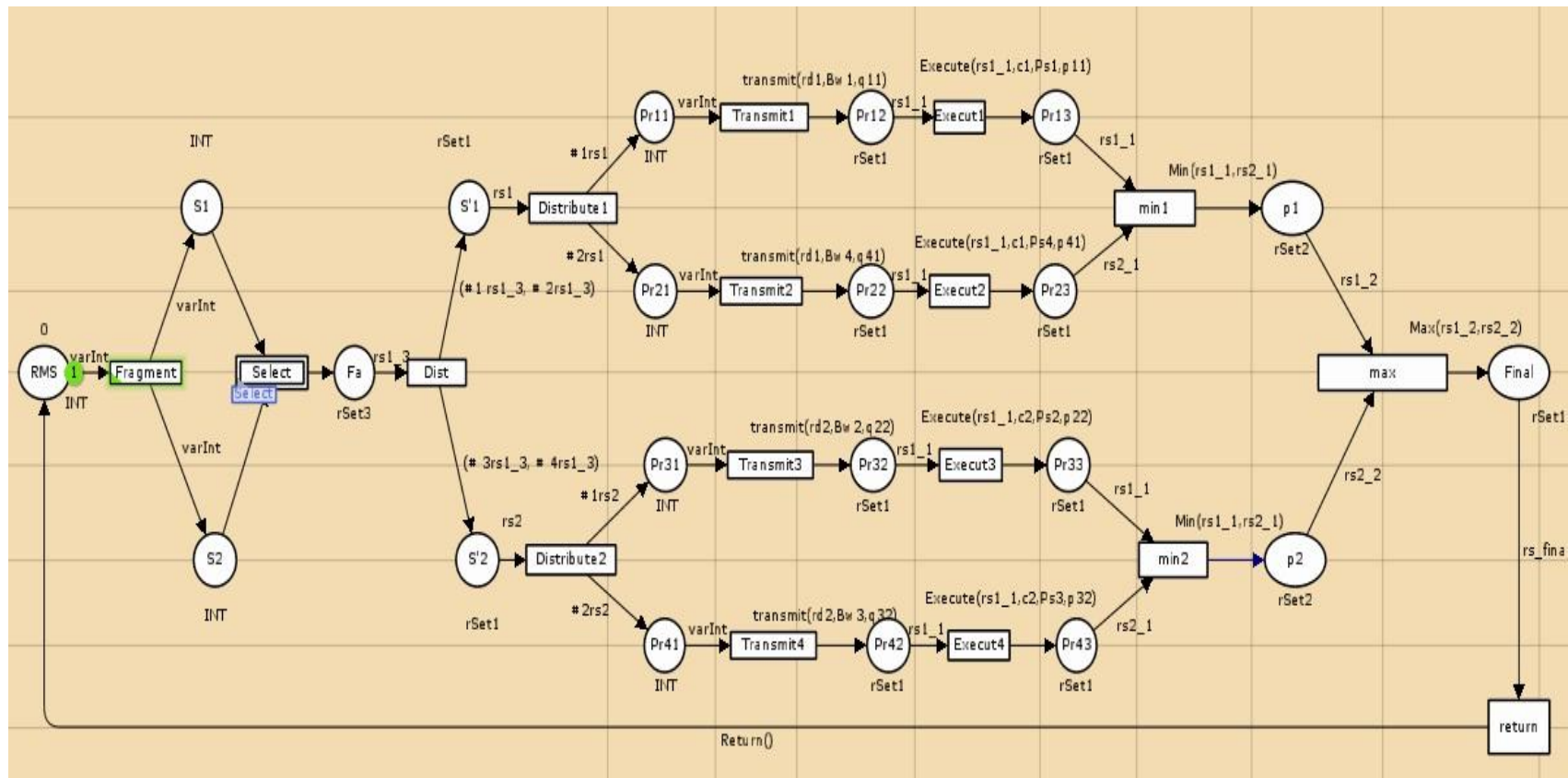

Fig3- The CPN model using CPN Tools

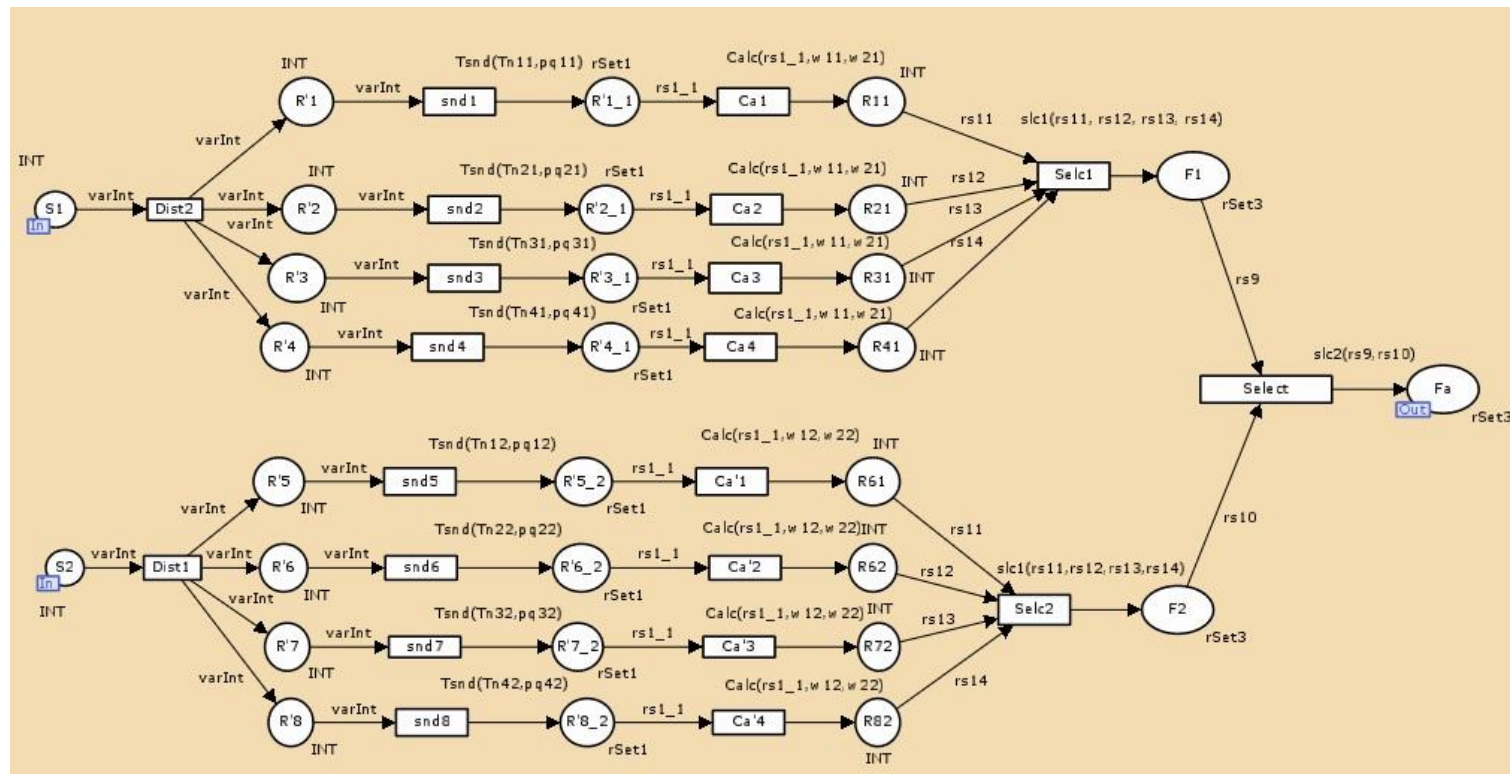

Fig4:The CPN model of the resources choice subnet using CPN Tools

In this section, 4 states are studied for evaluation of the reliability. Fig5 shows the reliability results achieved from the optimal choice of resources.

State1: subtask s1 chooses resources R2 and R4 and subtask s2 chooses resources R1 and R3 .

State2: subtask s1 chooses resources R1 and R4 and subtask $\mathrm{s} 2$ chooses resources R3 and R2 .

State3: subtask s1 chooses resources R1 and R3 and subtask s 2 chooses resources $\mathrm{R} 2$ and $\mathrm{R} 4$.
State4: subtask s1 chooses resources R4 and R3 and subtask s2 chooses resources R1 and R2 .

As it is seen in the graph, the proposed method has choices with higher reliability in higher $\mathrm{T}_{\text {reliable }} \mathrm{S}$.

Figure6 shows the reliability of previous system in the example[11]. Figure5 shows the reliability of the proposed method in the example. Figure5 includes the best states achieved for the system reliability, while the pervious model chose the resources randomly and every 4 states may occur for the system. 


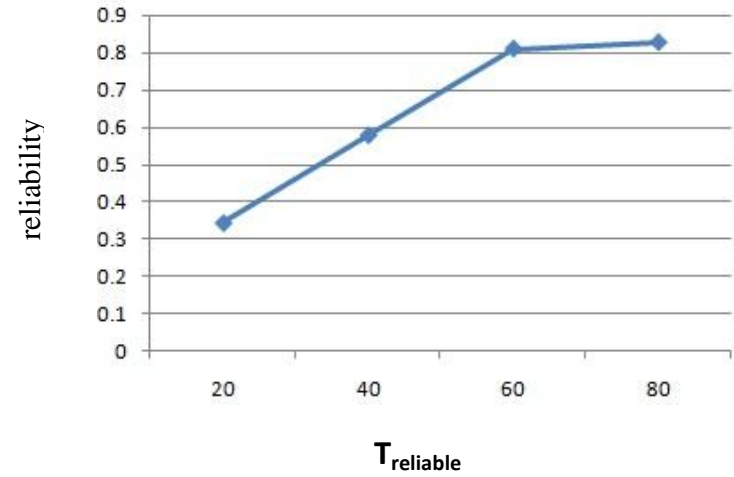

Fig5: reliability achieved from optimal selection of resource

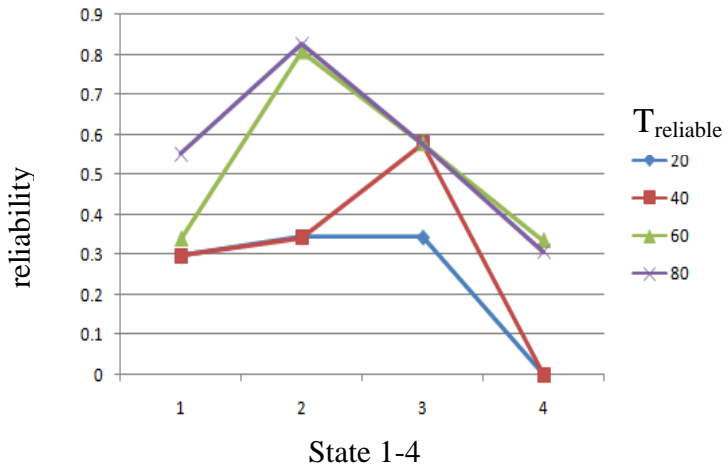

Fig6: reliability achieved from previous model

\section{CONCLUSION}

In this paper is presented a model for scheduling in the grid environment. In the model, the resources management system catches a task from the operator and divide it to subtasks and then give every subtask to a resource which has both more reliability and lower execution time. In the method, the operator can distinguish the importance of attributes for the system in order to choose the resources until the choice is accomplished considering the significances. The method is modeled through coloured petri nets. In addition, an example of proposed model for a sample grid environment is constructed and analysed using CPN Tools. The results demonstrated reliability improvement compared to the previous model.

\section{REFERENCES}

[1] I. Foster and C. Kesselman (editors), The Grid: Blueprint for a Future Computing Infrastructure, Morgan Kaufmann Publishers, 1999.

[2]R. Plestys, G. Vilutis, D. Sandonavicius, "The Measurement of Grid QoS Parameters", Proceeding of the ITI $2007,29^{\text {th }}$ Int.Conf.on Information Technology Interfaces, Cavtat,Croatia, Jun.2007 ,pp.25-28.
[3] X-He. Sun, M. Wu, "Quality of service of Grid Computing :Resource Sharing", The 6th International Conference on Grid and Cooperative Computing(GCC),2007.

[4] X. Wang, J. Luo, "Architecture of grid resource allocation management based on QoS", in: Grid and Cooperative Computing, Springer, Berlin, Heidelberg, 2004, pp. 81_88.

[5] Y-Sh. Dai, M. Xie, K.1. Poh, "Reliability of grid service system"s, Computers and Industrial Engineering,2006 pp.130_147.

[6] G. Levitin , Y-Sh. Dai , "Service reliability and performance in grid system with star topology", Reliability Engineering and System Safety 92, Elsevier, 2007 , pp. $40-46$.

[7]Y-Sh. Dai, G. Levitin, "Reliability and performance of tree-structured grid services", IEEE Transaction on Reliability ,June. 2006, pp. 337-349.

[8] Y-Sh. Dai, G. Levitin, K. Trivedi, "Performance and reliability of tree-structured grid service considering data dependence and failure correlation", IEEE Transaction on Computer July. 2007 ,pp. 925-936.

[9] G. Levitin, Y-Sh. Dai, H. Ben-Haim, "Reliability and performance of star topology grid service with precedence constraints on subtask execution" ,IEEE Transaction on Reliability Sept. 2006 ,pp. 507-515.

[10] Y-Sh. Dai, G. Levitin, X. Wang, “Optimal task partition and distribution in gridservice system with common cause failures", Future Generation Computer Systems,2007, pp.209_218.

[11]M.A. Azgomi, R. Entezari-Maleki, “Task scheduling modelling and reliability evaluation of grid services using coloured Petri nets", Future Generation Computer Systems 26 (2010) 1141-1150.

[12] K. Jensen, L.M. Kristensen, L. Wells, "Coloured Petri nets and CPN tools for modeling and validation of concurrent systems", International Journal on Software Tools for Technology Transfer (STTT), 2007,pp. 213_254.

[13] M.Meibody, M.Shojafa.M, S.barzegar, "A new Method on Resource Scheduling in grid systems based on Hierarchical Stochastic Petri net", 3rd International Conference on Computer and Electrical Engineering (ICCEE 2010),2010,pp.175-180.

[14] L.Li, Y. FangChun, "Modeling and Performance Analysis of a Priority-based Scheduling Schema in Service Grid", Proceeding of the 5th International Conference on Grid and Cooperative Computing(GCC'06),IEEE,2006,pp. 327-330.

[15] Y. Han, C. Jiang, Y. Fu, X. Luo, "Resource scheduling Algorithms for Grid Computing and Its Modeling and Analysis Using Petri Net" , Second International Workshop ,Springer, 2004,pp. 73-80

[16]S. Parsa, R. Entezari-Maleki, “ A queuing network model for minimizing the total makespan of computational grids", Computer and Electrical Engineering ,July 2012,pp.827-839.

[17] M. Jawad Asgharpour, Multiple Criteria Decision Marking, university of Tehran press, 2010. 